\title{
Utility of endoscopic ultrasound in hemorrhage from recurrent duodenal varices
}

\section{Shigenaga Matsui, Hiroshi Kashida, Masatoshi Kudo}

Kindai University Faculty of Medicine, Japan

A 69-year-old woman with medical history of endoscopic variceal ligation (EVL) of hemorrhagic duodenal varices at another hospital was transferred to our institution with hematemesis. Gastrointestinal endoscopy revealed a flat, scarred, post-EVL lesion in the second section of the duodenum (Fig. 1A). There were no other hemorrhagic lesions. Endoscopic ultrasound (EUS) revealed duodenal varices in the submucosal layer under the post-EVL lesion (Fig. 1B). Therefore, hemorrhage from recurrent duodenal varices was suspected. We performed endoscopic injection sclerotherapy (EIS) using a mixture of $1.5 \mathrm{~mL}$ N-butyl-2-cyanoacrylate (CA) and $0.5 \mathrm{~mL}$ Lipiodol at the post-EVL lesion (Fig. 1C). X-ray and computed tomography after EIS showed that the injected CA occupied the duodenal varices (Fig. 2A,B). No rebleeding was observed after EIS.

Management of hemorrhage from duodenal varices can be challenging because of the difficulty of treatment. Endosco-pic procedures, such as EIS with CA, EVL and clip, are less invasive compared to surgery or interventional radiology [1,2]. EUS can improve the detection and diagnosis of duodenal varices and collateral veins, and can facilitate the intravariceal injection of duodenal varices by techniques such as EIS. EUS is useful for revealing recurrence and helps in the management of duodenal varices.

Department of Gastroenterology and Hepatology, Kindai University Faculty of Medicine, Japan

Correspondence to: Shigenaga Matsui, MD, PhD, Department of Gastroenterology and Hepatology, Kindai University Faculty of Medicine, Osaka, Japan, e-mail: ma2i@med.kindai.ac.jp

\section{Conflict of Interest: None}

Received 29 April 2018; accepted 30 May 2018; published online 12 July 2018

DOI: https://doi.org/10.20524/aog.2018.0289
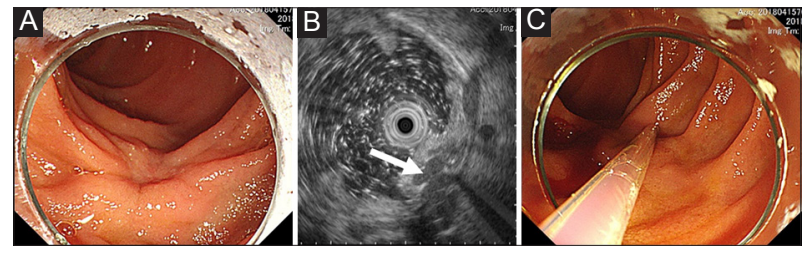

Figure 1 (A) Gastrointestinal endoscopy revealed a flat, scarred, post-endoscopic variceal ligation (EVL) lesion in the second section of the duodenum. (B) Endoscopic ultrasound revealed duodenal varices (arrow) in the submucosal layer under the post-EVL lesion. (C) Intravariceal injection of cyanoacrylate into the duodenum
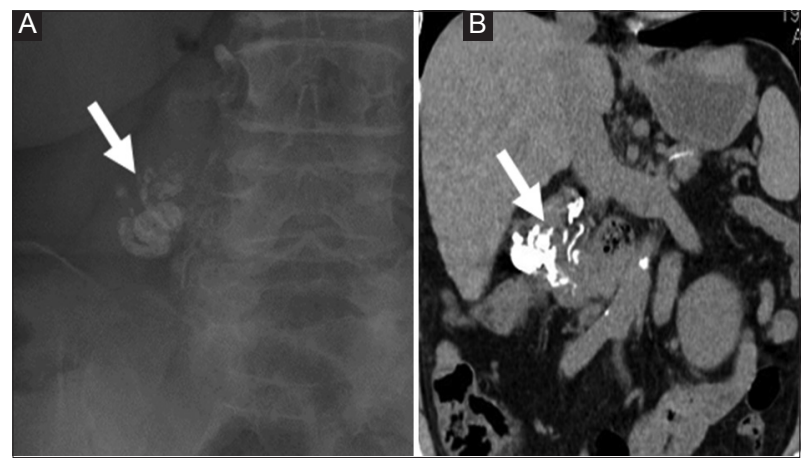

Figure 2 X-ray (A) and computed tomography (B) after endoscopic injection sclerotherapy showed that the injected N-butyl-2cyanoacrylate occupied the duodenal varices (arrow)

\section{References}

1. Matsui S, Kudo M, Ichikawa T, Okada M, Miyabe Y. The clinical characteristics, endoscopic treatment, and prognosis for patients presenting with duodenal varices. Hepatogastroenterology 2008;55:959-962.

2. Kakizaki S, Toyoda M, Ichikawa T, et al. Clinical characteristics and treatment for patients presenting with bleeding duodenal varices. Dig Endosc 2010;22:275-281. 